\title{
Population Growth and the Environment: Can We Eat the Cake and Have It?
}

\author{
SHMUEL AMIR \\ Division of Applied Physics, Soreq Nuclear Research Center, Yavne, 81800, Israel
}

(Received 16 November 1998)

\begin{abstract}
Continuous population growth and the concomitant maintenance of a growth sustaining environment are not in conflict only if the rates of both economic and biological processes are slowed down steadily. For this to happen, individual and social impatience-lowering adaptations should be adopted, and the capital of the system, both economic and natural, has to be redistributed among the system interacting components to result in an ever more complex organizational web. In addition, these factors have to corroborate with a third element, the incorporation of an arsenal of varied technological innovations that improves the use of existing stocks.
\end{abstract}

Keywords: Environmental costs, Period of production, Population growth, Sustainability, Technological progress, Thermodynamics-based theory of value

\section{INTRODUCTION}

Georgescu-Roegen (1971) states that the raison d'etre of the economic process is not merely materialistic, and its true output is not a physical flow of energy but the enjoyment of life. Furthermore, he is correct to observe that no definite, quantitative law seems to exist that relates common thermodynamic functions either to economic value or to its precursor, the enjoyment of life (GeorgescuRoegen, 1979). Most economists would agree that it is essential to concretize the concept of the enjoyment of life to discover the real sources of economic value. This task is still ahead of us. In the meantime one is safe concluding that as more people get an opportunity to enjoy life of a given quality, society entertains higher welfare. Thus, other things being equal, population growth is something to be cherished and encouraged. Georgescu-Roegen (1975) hesitates to accept this conclusion only because he does not believe that other things will remain the same with or without population growth. His reservation is based on the idea that the total enjoyment of life, given by the product of the number of individuals and the enjoyment entertained by a representative individual, is absolutely and irrevocably limited by some kind of a thermodynamic barrier.

Simon $(1980,1981)$ goes one step further. He views people rather than their satisfaction as the 
ultimate resource. Population growth is an autocatalytic process that promotes social welfare by positively feedbacking on itself. Truly, population growth creates pressures over resources, but the resulting scarcity is only temporal. It will be soon relieved because population growth generates both the means, the knowledge needed, and the right conditions to overcome scarcity successfully. Knowledge is created and prospers especially when the need for it exists, thus turning momentary scarcity into permanent abundance. According to Simon, historical trends show the human condition to be ever improving. Always there have been doomsayers around, but humankind has evolved, integrating a predisposition to deal effectively with resource scarcity. Physical limits, such as the size of the earth, and indeed the very concept of finitude, cannot be meaningful for determining the human condition. The ultimate determinant of the human condition is knowledge. The body of possible knowledge is not finite physically, and it has been growing faster than any stress. In sum, on balance people are creators rather than destroyers.

The IPAT model (Ehrlich and Holdren, 1971) represents an alternative viewpoint. According to this model, the impact I of the economy on the environment is the product of three terms: population $\mathrm{P}$, affluence $\mathrm{A}$ measured by the economic product per capita, and technology $\mathrm{T}$. The last term is an expression for the level of environmental damage inflicted by a given technology per unit of the economic product this technology generates. The model quantifies the idea that any economic process, consisting of the production, consumption, and exchange of goods and services in human societies, is carried out within an economy that constantly interacts with its environment. This interaction is embodied in diverse inflows and outflows that cross the boundaries of the economy. Many inflows are resources that are also needed by the environment for its own sustainability. Many outflows are harmful to the environment, and disposing them therein lowers the environment capability to generate future inflows. Thus, both flows generate social costs by inflicting damages on the environment and making it less conducive for future economic growth. This may affect directly future populations and their standard of living. Moreover, since some inflows may be absolutely essential for the survival of the human society, the deterioration of the environment may be detrimental for future generations.

Given the technology and economic well-being, the impact on the environment of the economic process is proportional to population size. No wonder, therefore, that many advocates of the IPAT model also support population stabilization (zero population growth) or even negative population growth as means to curb environmental damage (Ehrlich and Ehrlich, 1990). Two other ways to arrest the impact of human activities on the environment, limit affluence and improve the technology, are also possible. But they seem to be more challenging and difficult to achieve, at least for rates sufficiently potent to secure environmental sustainability (Bartlett, 1994). However, the relative efficacy of the various means is a matter of widely differing world views (Jolly, 1994). If population growth is really the engine that drives everything else, halting it should mitigate any harm done to the environment more effectively than working indirectly through secondary causes. This approach should also be less demanding in terms of its social hardship and economic resources. The conclusion may be totally different if human fertility responds voluntarily to environmental conditions (Easterlin, 1971). In particular, the best means will differ if population growth is the cart rather than the horse, and reducing it directly takes much time and works only in the very long run (Keyfitz, 1989).

Which means to take to reverse current trends of environmental decline and at what levels of activity to exercise them are questions of much importance for determining effective policies. These questions pertain to real-life problems that embrace every aspect of the human life: ethical, cultural, spiritual, and material (Emery, 1994). In recent years, much has been written to address both the professional audience and the general public on the policies involved and the consequences of any particular 
choice (Bongaarts, 1994; Goodland et al., 1994; Olson, 1994; Roush, 1994). Therefore, instead of recapitulating on the same issues in the present study, we would rather enlighten one, more fundamental, question of theoretical flavor: Is it really mandatory to halt population growth to achieve environmental sustainability? Put it in other words, we are concerned with the question of whether or not population growth and environmental sustainability are mutually exclusive goals, and which conditions, if any, allow them to coexist. We answer these questions in the context of the thermodynamics-based theory of value (Amir, 1994; 1995b).

The paper develops as follows. We first describe a typical economic process and lay out the conditions it must satisfy. We then study the relations between the economy and the environment and distinguish two possible frameworks. The first does not allow population growth and environmental sustainability to ever coexist. The second allows these two objectives to coexist if certain conditions are fulfilled. Finally, we define the appropriate conditions and discuss means to achieve them.

\section{THE ECONOMIC PROCESS}

The economy includes stocks of commodities and human agents who produce, consume, and exchange these commodities. Let there be in the economy $m$ commodities and $J$ activities. A commodity is any form of matter or energy. An activity, just like a chemical reaction, is a simplified method of transforming commodities into other commodities linearly. Whatever the system is doing is using activities (Lancaster, 1966; Muth, 1966), so that the term "activity" applies to the exchange as well as to the production and consumption of commodities and services. The economy is open thermodynamically, i.e. it exchanges matter and energy with its surroundings at the time that it operates activities.

Let $X_{j}$ be the level of the $j$ th activity, $b_{i j}$ be the amount of commodity $i$ produced by the $j$ th activity (per unit of time and activity), and $a_{i j}$ be the amount of commodity $i$ consumed by the $j$ th activity (per unit of time and activity). Denote by $Y\left(Y \in E^{m}\right)$ the flow of commodities associated with the economy. If $Y_{i}>0$ the $i$ th commodity flows out of the economy. If $Y_{i}<0$ the $i$ th commodity flows into the economy. Let $\mathrm{d} K\left(\mathrm{~d} K \in E^{m}\right)$ denote the change of the stocks of the economy during the period $\mathrm{d} t$. $\mathrm{d} K_{i}$ is positive for an increase in the $i$ th stock and is negative for a decrease. Thus, $K_{i}$ denotes the stock of the $i$ th commodity held within the boundaries of the economy. The definitions of the stocks and the boundaries are intertwined. For example, for our economy that contains the consumer sector within it, the stock vector must include components of human capital in addition to components of conventional capital. If some environmental resources have been internalized into the economy, their stocks have to be included in this vector as well.

Activities proceed in definite directions. In any activity products (outputs) are formed from reactants (inputs). We associate the inputs with the stocks $K_{t}$ and the inflows $-Y^{-}$during the period $\mathrm{d} t$. Hence,

$$
A X \mathrm{~d} t \leq-Y^{-} \mathrm{d} t+K_{t}
$$

Similarly, we associate the outputs with the final stocks $K_{t+\mathrm{d} t}$ and the outflows $Y^{+}$. Hence,

$$
B X \mathrm{~d} t \geq Y^{+} \mathrm{d} t+K_{t+\mathrm{d} t}
$$

where $A$ and $B$ - the input and output matrices, respectively - are $m \times J$ matrices and $X \in E_{+}^{J}$.

Recalling the definition of $\mathrm{d} K$ and dividing Eq. (2) by $\mathrm{d} t$, we derive a fundamental expression for the operation of the economy

$$
B X-A X \geq \mathrm{d} K / \mathrm{d} t+Y
$$

where $Y=Y^{+}+Y^{-}$.

To operate an activity, stocks of commodities are needed besides flows. Let $c_{i j}$ be the amount of commodity $i$ needed per unit of process $j$. We write:

$$
C X \leq K
$$

where $C$ is an $m \times J$ matrix. 
We are concerned with efficient systems. For this reason we assume that on the economy a welfare function $W(K, t)$ is defined that "guides" it to produce an efficient steady flow process. Neither this function nor its derivatives are observable variables of the economy. Moreover, welfare is not necessarily a real entity that exists independently of other forms of matter. Much like accounting money and "energy", it may be an abstract entity used to value other entities. It is important to bear this fact in mind on two counts. First, it is extremely difficult to overcome entrenched tradition that envisages welfare and energy to flow like matter (Mirowski, 1989). Only the flow of the latter is an observable variable of the economy. Second, matter and welfare are not unrelated either, because the theory postulates that the welfare function and its derivatives determine the observed flows. Any state function may become a welfare function, provided maximization of this function does not give rise to behavioral patterns that contradict observed behavior (Friedman, 1953).

Assume welfare is the accumulation of the following future "flows" of utility:

$$
\begin{aligned}
W\left(K_{0}, t_{0}\right)=\max _{Y}\{ & \int_{t_{0}}^{t_{1}} \mathrm{e}^{-\beta t} U(K, Y, t) \mathrm{d} t \\
& \left.+W\left(K_{1}, t_{1}\right)\right\}
\end{aligned}
$$

where $\beta$ is the rate of time preference.

In earlier studies (Amir, 1994; 1995b), we show that the fundamental equation that is satisfied by any efficient economic process is

$$
\begin{array}{r}
-(\dot{\alpha} p+\alpha \dot{p}) K=\max _{Y}\left\{\mathrm{e}^{-\beta t} U(K, Y, t)+\alpha p \dot{K}:\right. \\
\dot{K}=(B-A) X-Y ; C X=K\}
\end{array}
$$

where $W(K, t)$ is assumed to satisfy the condition $\delta W / \delta t=(\dot{\alpha} p+\alpha \dot{p}) K$.

The notation used in Eq. (6) is as follows: $\alpha(t)$ is a discount factor equal to $\alpha_{t}=\alpha_{0} \mathrm{e}^{-\beta t}$ and $\alpha(t) p(K, t)=\alpha_{t} p_{t}$ is a vector of the discounted real (welfare) values of the stocks, given by the derivatives of $W(K, t)$ with respect to $K$. Thus, $p(K, t)=p_{t}$ is a vector of the current real values.
The dot above a variable denotes the time derivative of the variable. Thus, $\dot{\alpha}=\mathrm{d} \alpha / \mathrm{d} t, \dot{K}=\mathrm{d} k / \mathrm{d} t$, and $\dot{p}=\mathrm{d} p / \mathrm{d} t=\delta p / \delta t+(\delta p / \delta K) \dot{K}$, where $\delta$ is the partial derivative operator.

One condition to be satisfied by an efficient process is

$$
\mathrm{e}^{-\beta t}(\delta U / \delta Y)=\delta W(K, t) / \delta K=\alpha(t) p(K, t) .
$$

Solving this condition for $Y$ as a function of $K$ and $t$ and substituting the solution back in Eq. (6), we derive an identity in $K$ as follows:

$$
\begin{aligned}
& (\dot{\alpha} p+\alpha \dot{p}) K+\alpha p \dot{K} \\
& \quad=(\dot{\alpha} p+\alpha \dot{p}) K+\alpha p\left\{(B-A) C^{-1} K-Y(K, t)\right\} \\
& \quad=-\mathrm{e}^{-\beta t} U(K, Y(K, t), t) .
\end{aligned}
$$

It is common to interpret the function $U(K, Y, t)$ as a sum of two components, say $U_{1}(K, Y, t)$ and $U_{2}(K, t)$ (Prigogine, 1961). The first component $U_{1}(K, Y, t)$ accounts for the change in $W(K, t)$ due to the inflows and outflows $Y$ and is equal to $p Y$. The second component $U_{2}(K, t)$ accounts for the change in $W(K, t)$ due to the process the economy is undergoing in isolation and is equal to $-((\dot{\alpha} p+\alpha \dot{p}) / \alpha) K-p(B-A) C^{-1} K$. In this process, the inputs $A X \mathrm{~d} t$ are transformed into the outputs $B X \mathrm{~d} t$. This process is directed, and it cannot be reversed naturally. The last condition is an expression of the second law of thermodynamics. It is incorporated into our description of the economy by claiming that $U_{2}(K, t)>0$ for any naturally possible process. Equation $\left(6^{\prime}\right)$ may now be decomposed into two equations as follows:

$$
\begin{gathered}
-\alpha p Y=-\mathrm{e}^{-\beta t} U_{1}(K, Y, t), \\
(\dot{\alpha} p+\alpha \dot{p}) K+\alpha p(B-A) C^{-1} K=-\mathrm{e}^{-\beta t} U_{2}(K, t) .
\end{gathered}
$$

The economy is in steady state if the vectors $\alpha p$, $K$, and $Y$, of discounted real values, stocks, and flows respectively, are time independent. Since in steady state $\alpha p$ and $K$ are time independent, the vectors $\dot{\alpha} p+\alpha \dot{p}$ and $\dot{K}$ are equal to zero. This implies that in steady state, the following 
equations are satisfied:

$$
\begin{aligned}
\alpha(t) p(K, t)(B-A) C^{-1} K & =\alpha(t) p(K, t) Y \\
& =-\mathrm{e}^{-\beta t} U_{1}(K, Y, t), \\
\alpha(t) p(K, t)(B-A) C^{-1} K & =-\mathrm{e}^{-\beta t} U_{2}(K, t) .
\end{aligned}
$$

These conditions are summed up as follows:

$$
\begin{aligned}
0 & =-\mathrm{e}^{-\beta t}\left\{U_{1}(K, Y, t)+U_{2}(K, t)\right\} \\
& =-\alpha p Y-\alpha p(A-B) C^{-1} K .
\end{aligned}
$$

In steady state, welfare is sustainable because the value of the flows, given by the first term on the right-hand side of Eq. (10), is equal to the value "consumed" in the process, given by the second term on the same side of the equation. Since $U_{2}(K, t)>0$ for any process whatsoever, in steady state $-U_{1}(K, Y, t)>0$ as well. The function $-\mathrm{e}^{-\beta t} U_{1}(K, Y, t)$ measures the net real value of the resources that are taken from the environment or disposed into it. Hence, the expression $-\mathrm{e}^{-\beta t} U_{1}(K, Y, t)=-\alpha p Y>0$ measures the environmental cost of maintaining the economy in steady state (Amir, 1995a).

The second condition to be satisfied by an efficient process is derived from Eq. $\left(6^{\prime}\right)$ by taking the derivatives of each side with respect to $K$. The result is

$$
\begin{aligned}
& \alpha p+\alpha \dot{p} \\
& \quad=-\left\{\mathrm{e}^{-\beta t}(\delta U / \delta K)+(\delta W / \delta K)(B-A) C^{-1}\right\} .
\end{aligned}
$$

In steady state each side of Eq. (11) should be zero. The condition $-\left\{\mathrm{e}^{-\beta t} U_{1}(K, Y, t)+\mathrm{e}^{-\beta t} U_{2}(K, t)\right\}=0$ is not sufficient to guarantee that an efficient steady state results. In an efficient steady state, the functions $-\mathrm{e}^{-\beta t} \mathrm{U}_{1}(K, Y, t)$ and $(\delta W / \delta K)(A-B) C^{-1} K$ should be tangential to each other at the solution point $K$, such that the former function lies entirely below the latter for any $K$ that differs from the solving $K$.

\section{THE ENVIRONMENTAL COST OF THE ECONOMIC PROCESS}

Let $K^{0}, X^{0}, Y^{0}$ be an optimal steady state scenario providing welfare $W^{0}$ and giving rise to the discounted real values $(\alpha p)^{0}$. This means that starting from $K^{0}$, the best plan is a steady state plan $Y^{0}$. Hence, the resulting scenario satisfies both the material equations $(B-A) X^{0}=Y^{0}$ and $C X^{0}=K^{0}$ and the value equations $\alpha \delta U / \delta Y=(\alpha p)^{0}$ and $\alpha \delta U /$ $\delta K=(\alpha p)^{0}(A-B) C^{-1}$. In view of Eqs. (10) and (11), the conditions $-\alpha\left\{U_{1}\left(K^{0}, Y^{0}, t\right)+U_{2}\left(K^{0}, t\right)\right\}=$ $-(\alpha p)^{0}\left\{Y^{0}+(A-B) C^{-1} K^{0}\right\}=0$ and $\dot{\alpha} p+\alpha \dot{p}=0$ respectively are also satisfied by this scenario. Consider the problem

$$
\min _{K}\left\{(\alpha p)^{0}(A-B) C^{-1} K: W(K) \geq W^{0}\right\}
$$

where $W(K)$ is the welfare derived from a steady state plan that starts with $K$. Assuming $(\alpha p)^{0}$ is independent of $K$, the first-order necessary conditions for a minimum are

$$
(\alpha p)^{0}(A-B) C^{-1}-\sigma \delta W(K, t) / \delta K \geq 0
$$

where $\sigma \geq 0$. The dimension of the Lagrange multiplier $\sigma$ is $1 /$ time, and it is equal to $\delta\left\{(\alpha p)^{0} \times\right.$ $\left.(A-B) C^{-1} K\right\} / \delta W(K, t)$. Thus, $\tau=1 / \sigma$ is the period of production (in economic terminology) or the turnover time (in physical terminology). It is the time required, on the average, for a unit of inflow to enter the economy, be processed there, and leave it as a unit of outflow.

In view of the definition of $(\alpha p)^{0}, K^{0}$ is the solution to problem (12). This implies that $\sigma^{0}=\sigma\left((\alpha p)^{0}, W^{0}\right)$ is a characteristic root and $(\alpha p)^{0}$ is a characteristic vector of $(A-B) C^{-1}$. If $\beta$ is free, it is equal to $\sigma^{0}$. In this case, $\delta U / \delta K=p^{0}(A-B) C^{-1}=\sigma^{0} p^{0}=\beta p^{0}=\dot{p}^{0}$. But if both $\beta$ and the matrices $A, B$, and $C$ are given, it cannot be guaranteed that $\beta=\sigma^{0}$. In this case, $\delta U / \delta K=p^{0}(A-B) C^{-1}=\sigma^{0} p^{0}<\beta p^{0}=\dot{p}^{0}$ (the case $\beta<\sigma^{0}$ is not possible as is shown later), and the economy is intertemporally inefficient. Lowering $\beta$ may start a modified scenario that will end up either in higher welfare or in lower environmental costs.

If we want to change the level of sustained welfare, we have to change the scenario and the corresponding discounted real values. Clearly, this will affect $\sigma(\alpha p, W)$ as well. Figure 1 shows the relation between the welfare to be sustained and the 


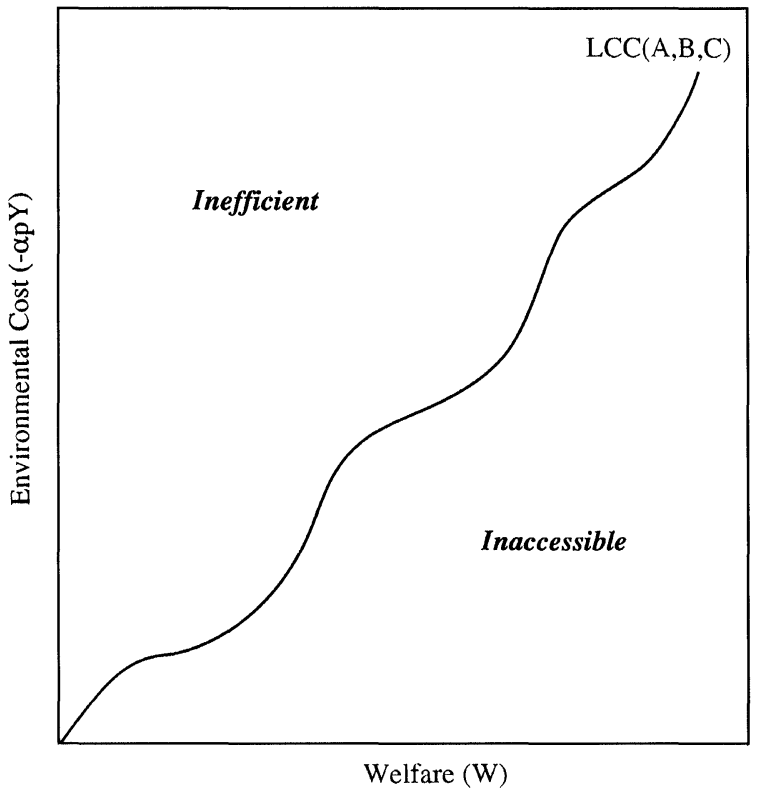

FIGURE 1 The environmental cost of sustainable welfare.

minimum environmental cost needed to sustain it, assuming the rate of time preference $\beta$ varies along the curve because it is equal to $\sigma(\alpha p, W)$. This curve may be called the Least Cost Curve (LCC), and it can be derived by solving problem (12) for different levels of welfare.

The LCC describes a collection of steady states that are derived by varying the welfare level, selecting the optimal stocks, and determining the resulting environmental costs. To be located on the LCC, the economy has to minimize the costs $\alpha p(A-B) C^{-1} K$ for every level of welfare. This is achieved by minimizing the speed of the production process $\sigma(\alpha p, W)$ (or equivalently by maximizing the production period $1 / \sigma(\alpha p, W))$. The economy is efficient and is located on the LCC when the endogenously determined discount rate $\sigma(\alpha p, W)$ is equal to the rate of time preference $\beta$. Whenever $\beta>\sigma(\alpha p, W)(\tau(\alpha p, W)>1 / \beta)$ the speed of the process is faster than the minimum possible and the process is less efficient than the maximum possible.

Let $W^{\prime \prime}>W^{\prime}$ be two welfare levels and let $e\left(\alpha p^{\prime \prime}, W^{\prime \prime}\right)$ and $e\left(\alpha p^{\prime}, W^{\prime}\right)$ be the respective minimum costs. We know that the following relations hold, $e\left(\alpha p^{\prime}, W^{\prime}\right)<e\left(\alpha p^{\prime \prime}, W^{\prime}\right)<e\left(\alpha p^{\prime \prime}, W^{\prime \prime}\right)$.
Therefore, an increase in $W(K)$ will increase $-\alpha p Y=e(\alpha p, W)$. This fact explains why the LCC in Fig. 1 is increasing.

The LCC separates the plane into two regions. The region above the curve is the region of inefficiency. Any system in steady state that spends more resources than the minimum needed to sustain its welfare be located in this region. The region below the curve is the region of inaccessibility. Given $A, B$, and $C$, which determine $\sigma(\alpha p, W)$, no system in steady state is located in this region because no system is able to support its welfare with less resources than the minimum needed. Both efficient and inefficient systems, not in steady state, however, may find themselves spending time in the inaccessible region. If the maximum value of the available resources is less than the minimum needed to sustain the welfare of the system in steady state, welfare will decrease, and a system within the region will necessarily move from one transient to another. This path may end either in extinction or in a steady state outside the inaccessible region. Similarly, in addition to inefficient systems in steady state, the region of inefficiency may inhabit other systems not in steady state. These systems necessarily move from one transient to another before they settle in a steady state.

Given the welfare sustained in steady state, the location of the system in the region of inefficiency depends on its realized period of production. If society is sovereign to decide on this matter, $\beta$ is dominant in its decision. The period of production will be $1 / \beta$, and the observed values of the elements of the matrices $A, B$, and $C$ will differ from the unobserved values that are to be used for determining technological efficiency. If the observed values of the elements are assumed to represent a system entertaining the highest possible efficiency, the realized period of production will be $1 / \sigma$. In this case, $\beta$ is not directly observable, and it cannot be determined independently of $\sigma$, the realized speed of the production process.

There are many ways to increase the efficiency of an inefficient system. Each alternative results in a mixture of an increase in welfare and a concomitant 
decrease of the incurred costs. It is important to note that this type of inefficiency should be cut irrespective of the welfare to be finally sustained. Once efficiency is achieved, society may face the harder issue of the welfare to be sustained and the environmental costs to be incurred. If the economy and its environment are viewed as an isolated system, the higher is the economy-derived welfare, the faster the environment loses its capacity to support the economy, i.e. higher environmental costs are involved. If the economy is to provide any welfare at all, some environmental costs must be incurred. How much is a matter of choice. If society is ready to decrease its sustained welfare, the loss of environmental resources can be decelerated.

This view needs some elaboration if we are not to be drawn into concluding that higher rates of environmental deterioration must be the immediate consequence of a higher sustained welfare. To examine this point, we compare two arrangements that are presented in Fig. 2(a) and (b). In Fig. 2(a) the economy is the system of concern and anything else is part of the overall surroundings. In Fig. 2(b) the inner part of the overall surroundings is separated from the remaining part, the outer surroundings. Let the current "flows" of value across these two boundaries be $U_{\mathrm{sl}}\left(K_{\mathrm{s}}, Y_{\mathrm{s}}, t\right)$ and $U_{\mathrm{e} 1}\left(K_{\mathrm{e}}, Y_{\mathrm{e}}, t\right)$ respectively.

From a methodological point of view, it is convenient to contain processes within systems holding the surroundings as an idle background. Consider Fig. 2(a), in this arrangement, the economy always satisfies Eq. $\left(6^{\prime}\right)$. According to this equation, if the economy is in steady state, the environment loses value, and the loss rate is $-\mathrm{e}^{-\beta t} U_{\mathrm{s} 1}\left(K_{\mathrm{s}}, Y_{\mathrm{s}}, t\right)=\mathrm{e}^{-\beta t} U_{\mathrm{s} 2}\left(K_{\mathrm{s}}, t\right)>0$. This is the only loss the environment incurs because no process is taking place within it. If the environment is unbounded, this loss does not imply a similar decrease of the total value of the environment. But if the economy and the environment together are isolated, as is usually assumed, this loss must represent a decrease in the value of the environment. This is shown as follows. Assume the environment is a system that is undergoing (a) Surroundings

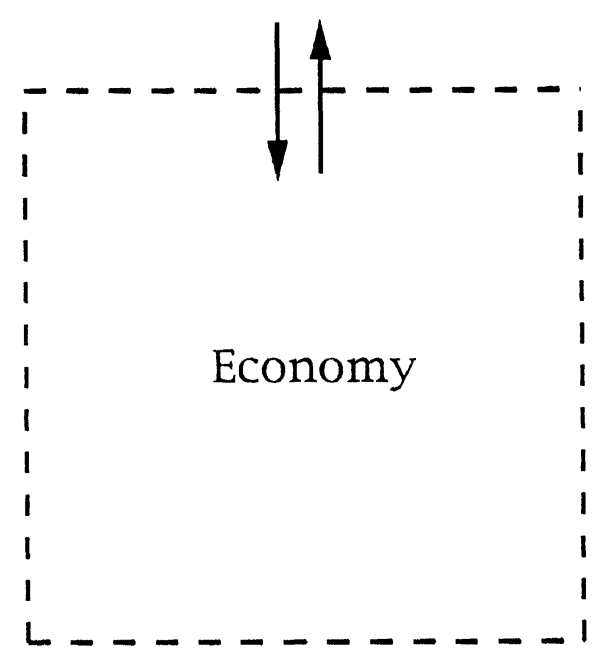

(b) Surroundings

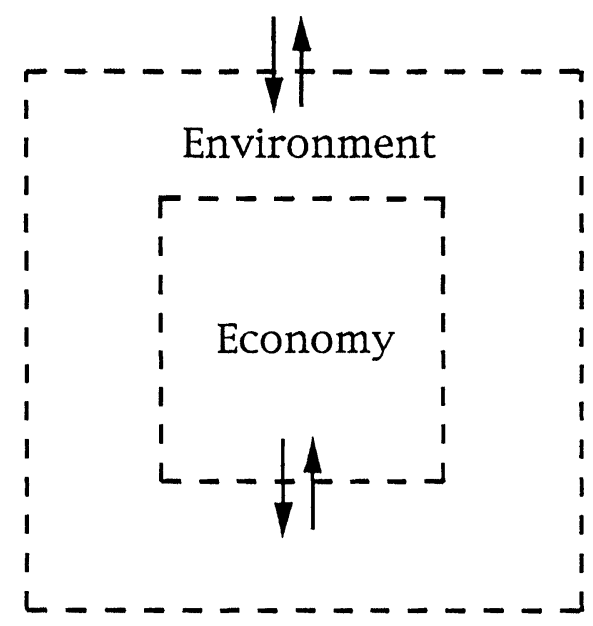

FIGURE 2 Two arrangements of the economy and its environment.

a process. This process must satisfy Eq. $\left(6^{\prime}\right)$. Since the environment interacts only with the economy, $\quad-\mathrm{e}^{-\beta t} U_{\mathrm{s} 1} \quad\left(K_{\mathrm{s}}, Y_{\mathrm{s}}, t\right)=\mathrm{e}^{-\beta t} U_{\mathrm{e} 1}\left(K_{\mathrm{e}}, Y_{\mathrm{e}}, t\right)>0$ and $\quad(\dot{\alpha} p+\alpha \dot{p}) K_{\mathrm{e}}+\alpha p\left\{\left(B_{\mathrm{e}}-A_{\mathrm{e}}\right) X_{\mathrm{e}}-Y_{\mathrm{e}}\right\}=$ $-\mathrm{e}^{-\beta t}\left\{U_{\mathrm{e} 1}\left(K_{\mathrm{e}}, Y_{\mathrm{e}}, t\right)+U_{\mathrm{e} 2}\left(K_{\mathrm{e}}, t\right)\right\}<0$. Hence, the economy is maintained in steady state or is growing at the direct expense of the deterioration of the 
environment. Clearly, in the context of isolated systems, there is little sense in discussing the validity of a process consisting of everlasting population growth together with environmental sustainability.

This case is not repeated in Fig. 2(b). When the region shown as environment, which was part of the overall surroundings in Fig. 2(a), undergoes a process, it has to satisfy Eq. $\left(6^{\prime}\right)$. Assuming the economy to be growing or at least to be in steady state so that $(\dot{\alpha} p+\alpha \dot{p}) K_{\mathrm{s}}+\alpha p\left\{\left(B_{\mathrm{s}}-A_{\mathrm{s}}\right) X_{\mathrm{s}}-\right.$ $\left.Y_{\mathrm{s}}\right\}=-\mathrm{e}^{-\beta t}\left\{U_{\mathrm{s} 1}\left(K_{\mathrm{s}}, Y_{\mathrm{s}}, t\right)+U_{\mathrm{s} 2}\left(K_{\mathrm{s}}, t\right)\right\} \geq 0$ does not force the environment to be deteriorating. For deterioration not to happen, the value of the flows across the outer boundaries of the environment must be greater than the combined "consumptive" flows of the economy and the environment. This is an immediate outcome of two conditions. The first, which applies to the environment, is $0<(\dot{\alpha} p+\alpha \dot{p}) K_{\mathrm{e}}+\alpha p\left\{\left(B_{\mathrm{e}}-A_{\mathrm{e}}\right) X_{\mathrm{e}}-\right.$ $\left.\left(Y_{\mathrm{e}}-Y_{\mathrm{s}}\right)\right\}=-\mathrm{e}^{\beta t}\left\{U_{\mathrm{el}}\left(K_{\mathrm{e}}, Y_{\mathrm{e}}, t\right)-U_{\mathrm{sl}}\left(K_{\mathrm{s}}, Y_{\mathrm{s}}, t\right)+\right.$ $\left.U_{\mathrm{e} 2}\left(K_{\mathrm{e}}, t\right)\right\}$. The second, which applies to the economy, is $-\mathrm{e}^{-\beta \mathrm{t}}\left\{U_{\mathrm{s} 1}\left(K_{\mathrm{s}}, Y_{\mathrm{s}}, t\right)+U_{\mathrm{s} 2}\left(K_{\mathrm{s}}, t\right)\right\}>0$. Combining the two conditions, we derive the desired outcome, which is $\mathrm{e}^{-\beta t} U_{\mathrm{el}}\left(K_{\mathrm{e}}, Y_{\mathrm{e}}, t\right)>-\mathrm{e}^{-\beta t} \times$ $\left\{U_{\mathrm{s} 1}\left(K_{\mathrm{s}}, Y_{\mathrm{s}}, t\right)+\mathrm{e}^{-\beta t} U_{\mathrm{e} 2}\left(K_{\mathrm{e}}, t\right)\right\}>\mathrm{e}^{-\beta t}\left\{U_{\mathrm{e} 2}\left(K_{\mathrm{e}}, t\right)+\right.$ $\left.U_{\mathrm{s} 2}\left(K_{\mathrm{s}}, t\right)\right\}$. This suggests that conditions may be found, in which population growth and sustainable environment are not conflicting. Clearly, for the whole exercise to make sense, the outer surroundings should be able to provide increasing levels of value in the form of $-\mathrm{e}^{-\beta t} U_{\mathrm{e} 1}\left(K_{\mathrm{e}}, Y_{\mathrm{e}}, t\right)$.

Figure 3 demonstrates how efficient steady states may be affected by technological progress. Given the technological rate of discount $\sigma(\alpha p, W)$, all technological opportunities have been exhausted once the rate of time preference $\beta$ is equal to $\sigma(\alpha p, W)$, i.e. the economy stays on the LCC. Further opportunities open up when the technological rate of discount decreases so that the period of production can be lengthened. To benefit from this opportunity, more capital should be accumulated and more roundabout and complex activities have to be pursued (Böhm-Bawerk, 1891). This implies that the rate of time preference $\beta$ has to decrease as well. When economic stocks and the ensued welfare are held fixed, lower environmental costs are sufficient to sustain this particular level of welfare. Since this argument applies to any level of welfare, the LCC shifts down. The economy becomes more efficient. Previously inaccessible states become available, and efficient states become inefficient. Given the lower rates and the new level of efficiency that they permit, either welfare may increase or environmental cost may decrease.

\section{THE ENVIRONMENTAL COST OF POPULATION GROWTH}

In this section, we assume that a process of population growth is represented by an increase over time of the vector $K$, i.e. $\dot{K}>0$. Equation $\left(6^{\prime}\right)$ and Figs. 2(b) and 3 provide both the general context and the specific conditions for the process of population growth to materialize. Since an increase in $K$ does not guarantee an increase in $W$, we assume explicitly that for the processes we are interested in both $K$ and $W$ increase. Hence, the lefthand side of Eq. $\left(6^{\prime}\right)$ is supposed to be positive and so is the right-hand side. This implies that $-\mathrm{e}^{-\beta t} U_{\mathrm{s} 1}\left(K_{\mathrm{s}}, Y_{\mathrm{s}}, t\right)>\mathrm{e}^{-\beta t} U_{\mathrm{s} 2}\left(K_{\mathrm{s}}, t\right)$, i.e. the value taken from the environment surpasses the value "consumed" by the economy. Since $K$ increases with time, so does the "consumed" value, $\mathrm{e}^{-\beta t} U_{\mathrm{s} 2}\left(K_{\mathrm{s}}, t\right)$. If either the technology or the rate of time preference remains the same, the increased "consumption" has to be matched by a corresponding increase of the supporting environmental costs. To sustain the environment, the value of the flows across the outer boundaries $-\mathrm{e}^{-\beta t} U_{\mathrm{e} 1}\left(K_{\mathrm{e}}, Y_{\mathrm{e}}, t\right)$ should increase as well. Assumed bounded, the flows $Y_{\mathrm{e}}$ cannot sustain a process of everlasting steady population growth.

Thus, the key to maintain an endless process of population growth in the face of bounded inflows $Y_{\mathrm{e}}$ is in the concomitant adoption of technological innovations, the reorganization of capital, and the decrease of the rate of time preference. If the increase of $\mathrm{e}^{-\beta t} U_{\mathrm{s} 2}\left(K_{\mathrm{s}}, t\right)$ with time, due to the increase of $K$, is more than compensated by simultaneous pressures to decrease this expression, or at least not 
to increase it as much as is needed to surpass $\mathrm{e}^{-\beta t} U_{\mathrm{e} 1}\left(K_{\mathrm{e}}, Y_{\mathrm{e}}, t\right)$, the growth of $K$ and $W$ may be sustained forever. Thus, while $K$ and $W$ are increasing, the economic process is slowed down, and the economy moves in Fig. 3 from higher to lower LCCs. These changes may go on forever because their limit, the reversible process, is never to be achieved. No real process can be reversible and proceed infinitely slowly. Hence, never would $\sigma(\alpha p, W)$ and $\mathrm{e}^{-\beta t} U_{\mathrm{s} 2}\left(K_{\mathrm{s}}, t\right)$ be zero, and never would the period of production $\tau=1 / \sigma(\alpha p, W)$ be infinite. The technological rate of discount $\sigma(\alpha p, W)$ is a positive number bounded away from zero and its inverse $\tau<\infty$. This means that always there will be some room for adopting future technological innovations and for further lowering of the rate of time preference. By accumulating knowledge, we occupy increasing parts of this room and stretch the bounds imposed by the flow $Y_{\mathrm{e}}$. Furthermore, although the flows $Y_{\mathrm{e}}$ may be bounded, their value $-\mathrm{e}^{-\beta t} U_{\mathrm{e} 1}\left(K_{\mathrm{e}}, Y_{\mathrm{e}}, t\right)$ is also affected by the technology and is subject to future increase due to innovations. It is important to note, however, that although the growth process may continue forever, materially the extent of growth is necessarily bounded. From a material point of view, the increasing population would at most approach the reversible limit asymptotically.

In Fig. 4, we describe a complex process of population growth. In this process, initially the rate of technological changes more than counterbalances the increase in "consumption" $\mathrm{e}^{-\beta t} U_{\mathrm{s} 2} \times$ $\left(K_{\mathrm{s}}, t\right)+\mathrm{e}^{-\beta t} U_{\mathrm{e} 2}\left(K_{\mathrm{e}}, t\right)$ due to the growing population and natural capital respectively. This results in a steady, or even an accelerated, growth phase. Later, the rate of technological changes is not sufficient to counteract the increased "consumption". Nevertheless, growth continues because the net flow of value into the system $-\mathrm{e}^{-\beta t} U_{\mathrm{e} 1}\left(K_{\mathrm{e}}, Y_{\mathrm{e}}, t\right)$ also increases when knowledge, both technical and genetical, accumulates. This is the asymptotic phase where both the net flow across the system boundaries and "consumption" increase towards their reversible limit. Since their difference is the change in total welfare, as long as this difference is positive,

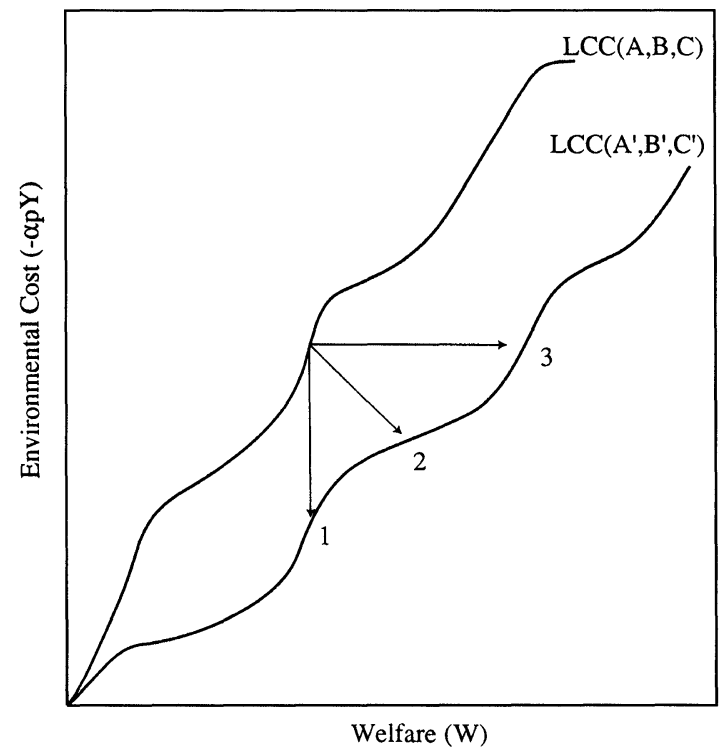

FIGURE 3 The effect of technological progress on the environmental cost.
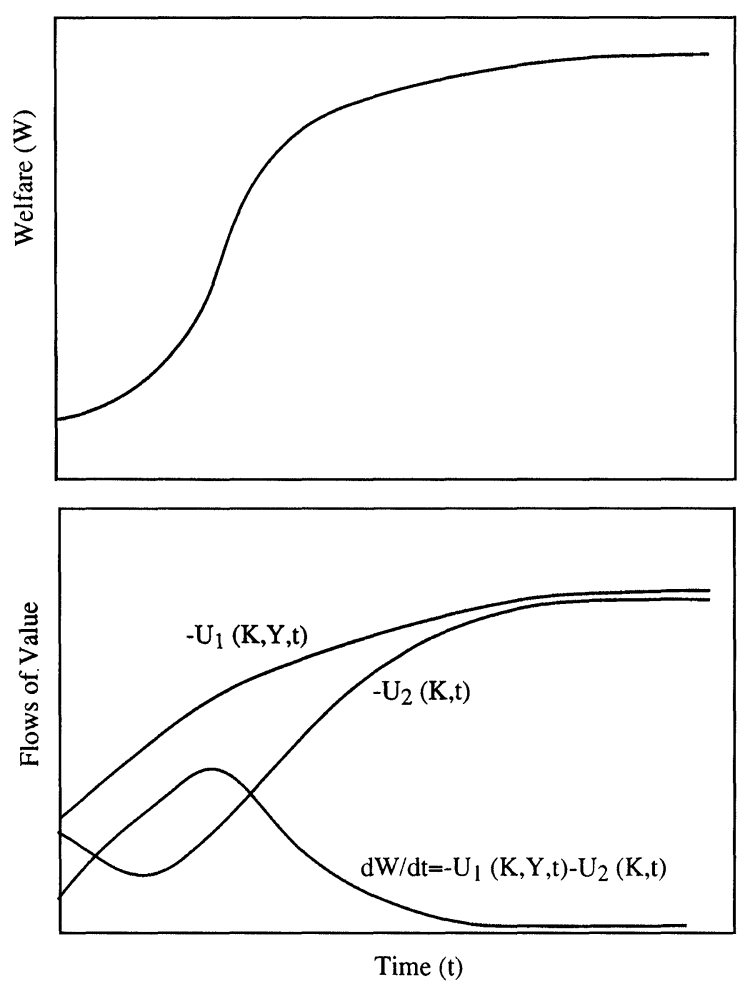

FIGURE 4 Welfare, environmental costs, and the "consumption" of value during an asymptotic process of population growth. 
total welfare also increases asymptotically. According to this description, doomsday will be here not because the process of population growth bounds to harm the environment but because someday the external source of value may be extinguished.

\section{DISCUSSION}

Numerous efforts have been made to arrest the decline in the quality of the environment. Nevertheless, the accelerated globalization of many environmental stresses is indisputable. The role of the economic process in the ongoing deterioration of the environment is also a common knowledge. In view of the number of interfaces across which the economy and the environment interact daily and hourly, this tendency of the economy to inflict damage on the environment is hardly surprising. But the question still stands: Are we to conclude from this state of affairs that population growth and the concomitant growth of the economy bound to lead to environmental stress and resource scarcity?

Simon $(1980,1981)$ claims the answer to this question is negative. He is a great believer in the role of knowledge and in its ability to disconnect the depressing circle whereby scarcity breeds poverty, population growth, and further scarcity. Simon envisions a society that by its own growth and in response to the ensued resource scarcity generates sufficient knowledge to relieve any stress on the environment and to create room for future growth. According to Simon, knowledge is the great engine that turns scarcity into affluence.

This view has been supported by many growth advocates but attacked by many environmentalists. Both supporters and opposers have presented data to justify their positions. However, the main question is not to be solved on facts alone if only because always it would be possible to find data to back up either position. Historical trends, no matter of which kind, are in no place to undermine fears, founded or unfounded, of drawing a steadily growing population into extinction because some life- supporting conditions collapsed after having been outstretched over their absolute limits.

The view that growth is steady is at the heart of the discussion. Both growth advocates and doomsayers hold this view when they speak about growth and the environment. This is a regrettable situation that blinds both groups to other possibilities. By envisioning resources to be perfectly substitutable, the growth advocates, particularly of the neoclassical school in economics, belittle the role of bounds. In their turn, the doomsayers overemphasize the role of bounds, assuming them to be always effective, whether growth is steady or not. This position is particularly evident with the limit to growth school (Meadows et al., 1972) and the IPAT model.

In this paper, we show Simon's position to be sound. It is reasonable to expect the creation of knowledge to ease environmental pressures and nurture further growth provided the resulting growth is asymptotic rather than steady. A process of growth may last forever, but if endless it cannot be steady. No law forces population growth to end in demise. But if neither the environment nor the population is to collapse, the ever-growing population should approach a limit. Since this condition is missing from Simon's analysis, his controversy with the doomsayers could not be resolved one way or another.

While the paper demonstrates the possibility for a growing population and a sustaining environment to coexist, nowhere is it claimed that exercising growth with environmental safety is easily practiced. To achieve both objectives may be extremely difficult, as the current state of the environment indicates. However, this is beyond the point of the present paper, which rather is concerned with a theoretical dilemma - that is, whether or not population growth and a sustained environment are in principle compatible.

For these objectives to agree both the general context and the specific conditions should be appropriate. No amount of technological knowhow can cut the "consumption" of value completely. To change their value, resources need participate in a process that necessarily "consumes" value. 
Hence, the decrease of global welfare with time in an isolated system is irrevocable. This decrease may be moderated by the invention and timely adoption of advanced technologies, but it cannot be avoided. The most one can aspire to is that the necessary decrease of the environment-derived welfare be accompanied by an asymptotic increase of the economy-derived welfare before the imperative doomsday is reached. Only if the inconceivable happens and the theoretical limit of the reversible process is reached before the environment-derived welfare is exhausted, doomsday is postponed indefinitely.

Barring this fantasy, we must consider the possibility of population growth futures in contexts other than the isolated system, e.g. either for open or for closed systems that allow at least energy flows across their boundaries. Viewed from this perspective, the economy and the natural ecosystem are two interacting subsystems of a larger system, say the Earth and its atmosphere. A necessary condition for human survival is the availability of sufficient organic stocks somewhere on Earth. These stocks find their own living either in natural ecosystems or in human controlled biosystems. For survival these systems depend on the surroundings, the rest of the solar system, in a direct and immediate way. Although doomsday, again, is imperative, it is very far away. This fact forces us to focus on the welfarecontributing capacity of the Earth rather than on that of the entire solar system. A meaningful question would be how we maximize welfare over time, given our present endowments of economic and ecological stocks and the available solar power. In this context, aggregate welfare - that is, the sum of the economy-derived and the natural ecosystemderived welfare - will increase as long as the value "transported" into the system from its environment surpasses the value "consumed" within the system and dissipated. In a closed system, this increase is accompanied by redistribution of the various stocks. The fraction of the stocks that participate actively in the economic and ecological processes increases, and more value is "consumed" per unit of time. This process can continue as long as the increased "consumption" of value net of possible savings due to the introduction of more efficient processes is matched by an increased utilization of solar power. Solar power is far from being fully utilized but is finite. Its limit can be conceivably reached. However, there always will be some room for more efficient processes because the reversible process, which is the most efficient, cannot be realized. As long as the rate of adoption of more efficient technologies is sufficiently fast to counterbalance the increasing "consumption" of value caused by the redistribution of the stocks, aggregate welfare may increase, approaching an asymptotic limit. Along this future, the rate of time preference decreases and the period of production increases. In addition, due to the dependency of the economy on the ecosystem, both the economy-derived and the ecosystem-derived welfare will increase in accordance with the thermodynamics-based theory of value.

\section{References}

Amir, S. 1994. The role of thermodynamics in the analysis of economic and ecological systems. Ecological Economics, 10, $125-142$.

Amir, S. 1995a. The environmental cost of sustainable welfare. Ecological Economics, 13, 27-41.

Amir, S. 1995b. Welfare maximization in economic theory: Another viewpoint. Structural Change and Economic Dynamics, 6, 359-376.

Bartlett, A.A. 1994. Reflections on sustainability, population growth, and the environment. Population and Environment, 16, 5-35.

Böhm-Bawerk, E.V. 1891. The Positive Theory of Capital. G.E. Stechert, NY.

Bongaarts, J. 1994. Population policy options in the developing world. Science, 263, 771-776.

Easterlin, R.A. 1971. Does human fertility adjust to the environment? American Economic Review, 61, 399-407.

Ehrlich, P.R. and Holdren, J.P. 1971. The impact of population growth. Science, 171, 1212-1217.

Ehrlich, P.R. and Ehrlich, A.H. 1990. The Population Explosion. Simon and Schuster, New York, NY.

Emery, K.O. 1994. Uncontrolled growth of human populations, geological background, and future prospects. Population and Environment, 15, 303-327.

Friedman, M. 1953. Essays in Positive Economics. University of Chicago Press, Chicago, IL.

Georgescu-Roegen, N. 1971. The Entropy Law and the Economic Process. Harvard University Press, Cambridge, MA.

Georgescu-Roegen, N. 1975. Energy and economic myths. Southern Economic Journal, 41, 347-381.

Georgescu-Roegen, N. 1979. Energy analysis and economic valuation. Southern Economic Journal, 45, 1023-1058. 
Goodland, R., Daly, H. and Kellenberg, J. 1994. Burden sharing in the transition to environmental sustainability. Futures, 26, $146-155$.

Jolly, C.L. 1994. Four theories of population change and the environment. Population and Environment, 16, 61-90.

Keyfitz, N. 1989. The growing human population. Scientific American, 258, 71-78.

Lancaster, K.J. 1966. A new approach to consumer theory. J. Pol. Econ., 74, 132-157.

Meadows, D.H., Meadows, D.L., Randers, J. and Behrens, W.W. III. 1972. The Limits to Growth. New American Library, New York, NY.

Mirowski, P. 1989. More Heat than Light: Economics as Social Physics, Physics as Nature's Economics. Cambridge University press, Cambridge, England.
Muth, R.F. 1966. Household production and consumer demand functions. Econometrica, 34, 699-708.

Olson, R.L. 1994. Alternative image of sustainable future. Futures, 26, 156-169.

Prigogine, I. 1961. Introduction to Thermodynamics of Irreversible Processes (2nd edn.). J. Wiley \& Sons, New York, NY.

Roush, W. 1994. Population: the view from Cairo. Science, 265 1164-1167.

Simon, J.L. 1980. Resources, population environment: an oversupply of false bad news. Science, 208, 1431-1437.

Simon, J.L. 1981. The Ultimate Resource: Population, Resources, Environment: A Different View. Princeton University Press, Princeton, $\mathrm{NJ}$. 


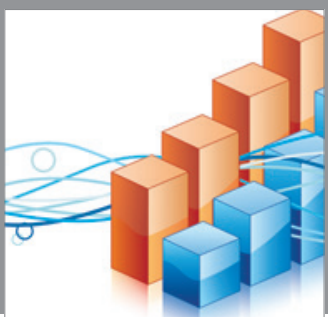

Advances in

Operations Research

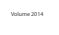

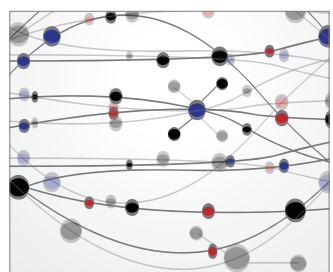

\section{The Scientific} World Journal
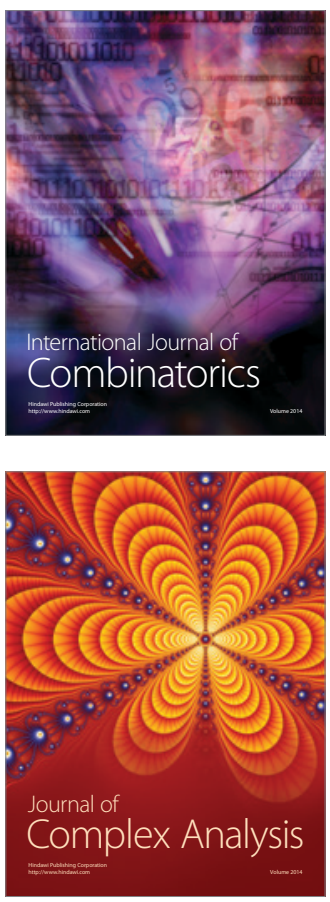

International Journal of

Mathematics and

Mathematical

Sciences
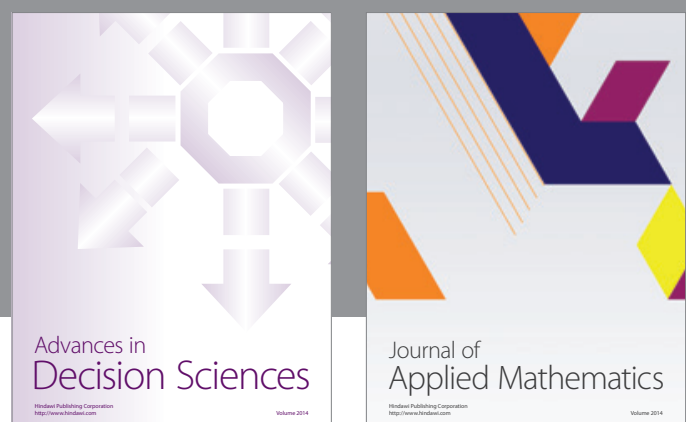

Journal of

Applied Mathematics
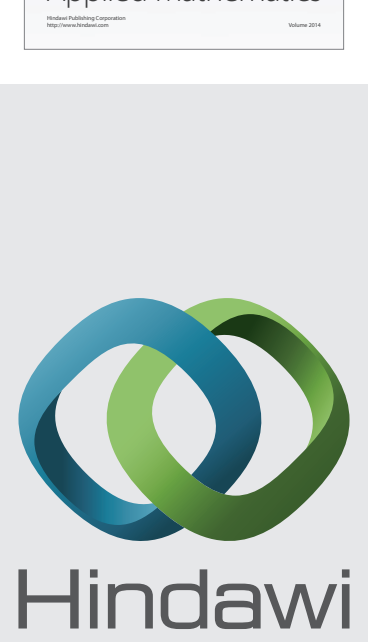

Submit your manuscripts at http://www.hindawi.com
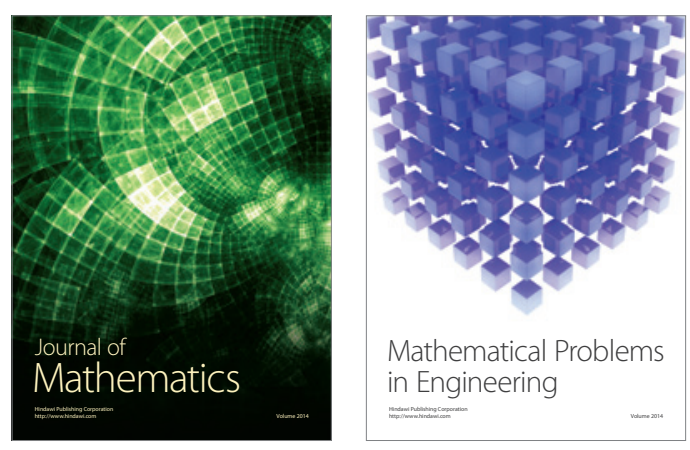

Mathematical Problems in Engineering
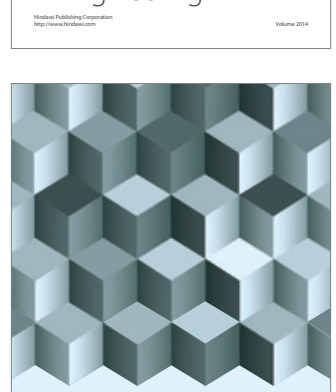

Journal of

Function Spaces
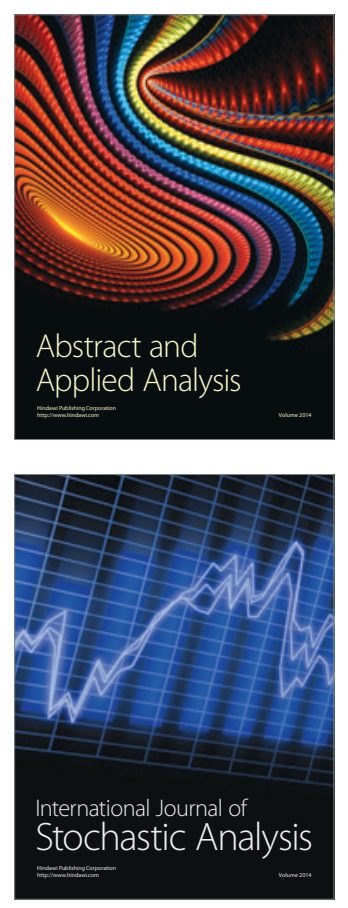

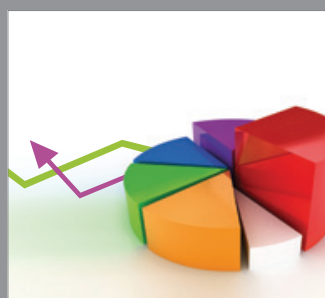

ournal of

Probability and Statistics

Promensencen
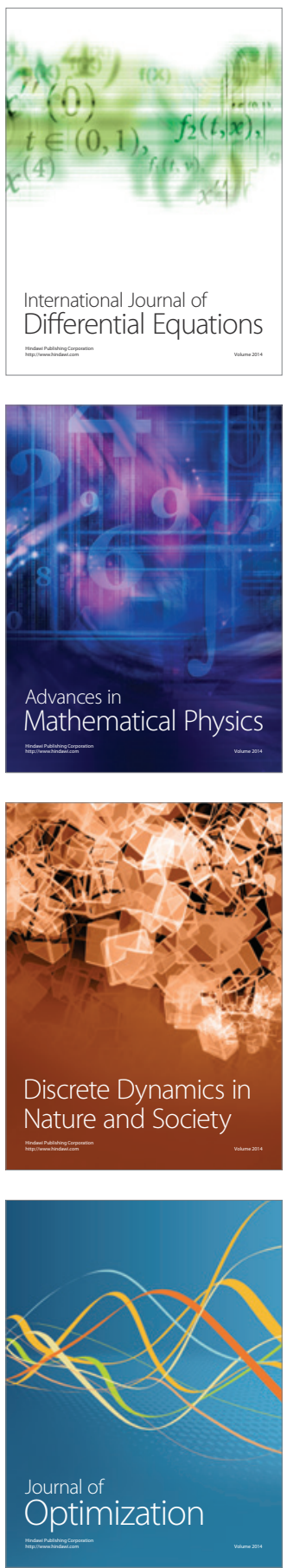